\title{
Electronic Holography
}

\author{
Chung J. Kuo \\ National Chung Cheng University \\ Department of Electrical Engineering \\ Media Processing Laboratories \\ Chiayi, Taiwan 62107 \\ E-mail:kuo@ee.ccu.edu.tw
}

Due to technological progress, holographic information (or interference patterns) can now be available through computer precalculation or captured by intensity recording devices. On the other hand, the resolution and response times of display devices are increasing such that the real-time holographic pattern can be shown on a display device. These technologies push the advent of "electronic holography.' 'Generally speaking, electronic holography represents the capture and reproduction of 3-D holographic information through the use of electronic (or electronic-driven) devices. Three-dimensional holographic information can be captured by an $\mathrm{O} / \mathrm{E}$ device or obtained through a computer. The $3-\mathrm{D}$ object can then be reconstructed by using electro-, acousto-, or magnetooptics devices or computer peripherals.

This technique has significant impact on every field related to holographic applications, and will revolutionize the display and printing industry. In addition, it can also be used in metrology applications. Apparently, it will also be a key technique in the consumer market. The applications (with more on the way) of electronic holography include (a) 3-D display, movie, and printing; (b) real-time metrology and calibration; (c) real-time information processing and computing; and (d) microscopic applications.

Many researchers around the world are interested in this topic. Some promising research results appear in the annual conference on practical holography, which is sponsored by SPIE and chaired by Professor S. A. Benton. The purpose of this special section is thus to report the current status of this research area, highlight its possible research directions, and show its impact on our society.

Many invitations were sent to potential authors all over the world. Eleven proposals were received and six of them have been accepted for publication. The following is a brief description of these six papers. One paper deals with holographic bandwidth compression using spatial subsampling, while another paper is concerned with nonuniformly sampled computer-generated holograms. These two papers concentrate on the studies of holographic fringe patterns, and they use acousto-optics devices for reconstructing them. A third paper discusses improvements to electro-holographic display using liquid crystal devices to shorten the viewing distance with both-eye observation. They replace the acousto-optics device (used in the first two papers) by the liquid crystal device. Another paper studies twin-image noise in optical scanning holography, which generates holographic information by 2-D active optical scanning. One paper proposes a parallel exposure system for high-speed recording of full-parallax holographic stereograms. The authors solve the lengthy exposure problem in a full-parallax holographic stereogram. Also, in another paper, the authors propose a parallax images capture system for (holographic) stereographic display.

I am grateful to the authors who submitted their work to this special section. I also acknowledge the precious and timely comments from the anonymous reviewers to improve the quality of this special section. Finally, I offer many thanks to Dr. Brian J. Thompson for giving me this opportunity to serve the readers of Optical Engineering. The patience and kindly help of Dr. Thompson and his Optical Engineering staff during my editoral process was truly appreciated.

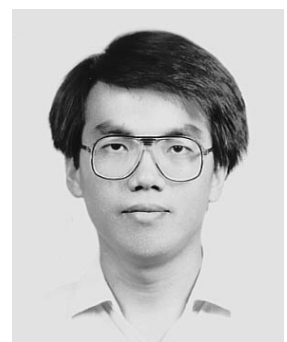

Chung J. Kuo received the BS and MS degrees in power mechanical engineering from National Tsing Hua University, Taiwan, in 1982 and 1984, respectively, and a PhD degree in electrical engineering (EE) from Michigan State University (MSU) in 1990. He was an instructor at Army Ordnance School from 1985 to 1986, and a visiting scientist at OptoElectronics \& System Lab, Industrial Technology Research Institute, during the summer of 1991. Dr. Kuo has been an associate professor of EE at National Chung Cheng University (NCCU) since 1990, and an adjunct associate professor of EE at National Cheng Kung University since 1991. His research interests include image processing, optical information processing/computing, and information theory, and he has had numerous publications in these areas. He is the director of the Media Processing Laboratories at NCCU. Dr Kuo received the Best Engineering Paper Award from Taiwan's Computer Society in 1991 and the Outstanding Academic Achievement Award from MSU in 1987. He is an associate editor for IEEE Signal Processing, a guest editor for two special sections of Optical Engineering, and an invited speaker and program committee member for several international/local conferences. He also serves as Secretary of SPIE, Taiwan Chapter. Dr. Kuo is a member of Phi Kappa Phi, Phi Beta Delta, IEEE, OSA, and SPIE. He is also listed in Who's Who in the World, 1995-1996. 